\title{
Lnm_Militärgasoto- doos.
}

Die Zeit, welcher die sogenannten Tactica Leonis angehören, ist

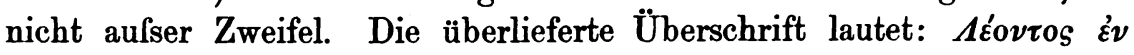

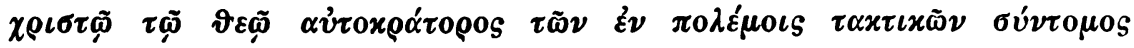
$\pi \alpha \varrho \alpha ́ \delta 0 \sigma \iota s$. Müssen wir darnach annehmen, dafs sie von einem Kaiser Leo herrühren, so fragt es sich doch, welcher von den mehreren Kaisern dieses Namens gemeint ist.

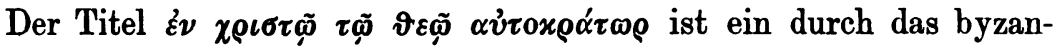
tinische Mittelalter hindurch gebräuchlicher. Die Sprache der $\tau \alpha x \tau \iota x \grave{\alpha}$ ist das byzantinische „Hochgriechisch“, wenn ein solcher Ausdruck erlaubt ist, und giebt ebenso wenig einen Fingerzeig für Lösung des Problems: es wäre denn in dem Gebrauche gewisser Wörter als Aus-

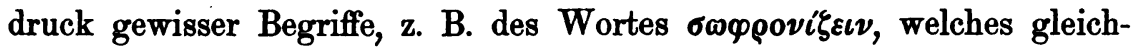

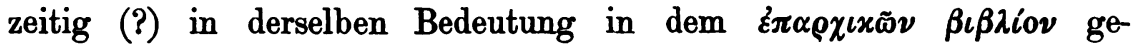
braucht wird.

Dagegen scheinen zwei Stellen des Proömium bestimmt auf den Kaiser Leo, der den Beinamen $\delta$ бopòs erhalten hat, den Sohn des Macedoniers Basilius, hinzuweisen. Der Kaiser sagt hier, er wolle eine

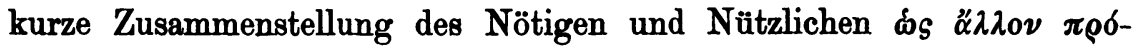
$\chi \varepsilon \iota \rho \circ \nu \nu \delta \mu o \nu$ geben, und wiederholt am Schlusse, dafs er die $\pi \alpha \rho \circ \tilde{\sigma} \sigma \alpha$

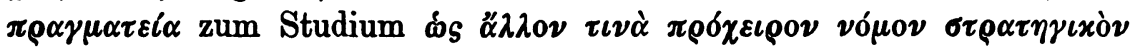
empfehle. Es liegt nahe hierin einen Hinweis auf das kleine Rechtsbuch zu sehen, welches von dem Kaiser Basilius dem Macedonier und seinen Söhnen Konstantin und Leo, und zwar nicht ohne besondere Beteiligung des Letzteren, publicirt worden ist, und welches ich

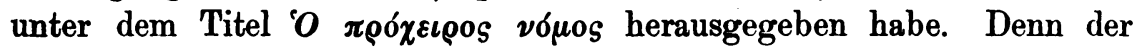
Kaiser und seine Söhne gebrauchen im Proömium desselben $\S 1$ die

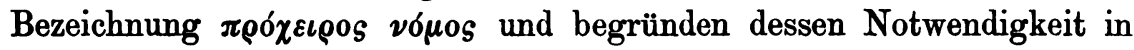
ganz gleicher Weise wie die der Tactica.

Indessen für ihr eigenes Rechtsbuch gebrauchen sie aufserdem in § 2. 4 die Ausdrücke $\pi \rho \alpha \gamma \mu \alpha \tau \varepsilon i \alpha$ und $\varepsilon \gamma \chi \varepsilon \iota \rho i \delta \iota s$, und zwar letzteren in bewufstem Gegensatz zu dem älteren $\varepsilon \gamma x \varepsilon\llcorner\rho i \delta \iota o s$, welcher von den Bilderstürmern Leo und Konstantin promulgirt worden und unter dem 


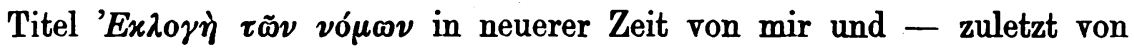
Monferratus, Athen 1889 - herausgegeben ist.

Wenn hiernach die angeführten Stellen nichts für Leo den Weisen als Verfasser der Tactica beweisen, so spricht gegen diese Urheberschaft noch insbesondere, dafs dieser Leo sich kaum als Urheber eines Rechtsbuchs hätte erwähnen können, welches bereits von seinem Vater promulgirt worden war: in anderen Fällen liebt er viëlmehr die gesetz-

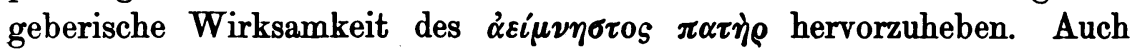
würde Leo's Sohn, Konstantin der Purpurgeborene, von dem eine Umarbeitung der Tactica erhalten ist, eine solche nach wenigen Jahren nicht für nötig gehalten haben, oder doch, wenn Aelianus und wo von ihm

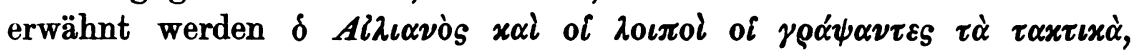
des Vaters besonders gedacht haben. Dafs dies nicht geschehen ist, spricht entschieden gegen eine Bearbeitung der Tactica durch Leo den Weisen. Sehen wir nun aber von Leo dem Weisen ab und sehen wir uns nach einem anderen Kaiser Leo um, der gesagt haben könnte, dals

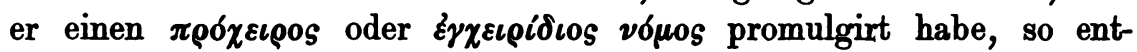

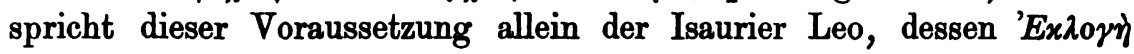
$\tau \tilde{\omega} \nu \nu \delta \mu \omega \nu$ eine so bedeutende Stellung in der Geschichte des byzantinischen Rechts einnimmt.

Dafs dieser Kaiser Leo der Urheber ist, wird nun auch durch andere Anzeichen wahrscheinlich gemacht.

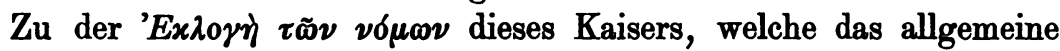

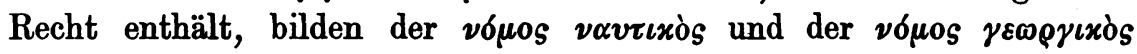
gewissermalsen Anhänge, die das für einzelne Interessentenkreise gültige Recht zusammenfassen: Einen ähnlichen für das Heer bestimmten An-

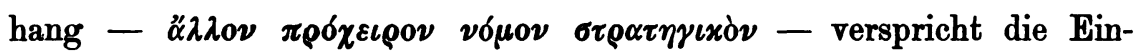
leitung zu den Tactica und bemerkt, dals derselbe auch die gesetzlichen Strafbestimmungen für die Vergehen der Soldaten - $\tau \grave{\alpha} \quad x \varepsilon i \mu \varepsilon \varepsilon \nu \alpha$ $\dot{\varepsilon} \pi \iota \tau i \mu \iota \alpha$ - enthalten solle.

Dies geschieht denn auch in Kap. VIII, welches die Überschrift $\pi \varepsilon \rho i \sigma \tau \rho \alpha \tau \iota \omega \tau \iota x \tilde{\nu} \nu$. $z \pi \iota \tau \iota \mu i \omega \nu$ führt. Betrachtet man dies Kapitel genauer, so findet man, dafs es in zwei Teile zerfällt. Der erste Teil

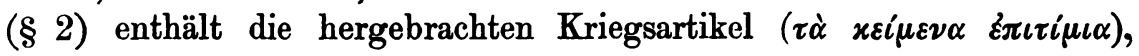
welche den Truppen bei den regelmäfsigen Aufstellungen vorzulesen sind. Daran reihen sich in einem zweiten Teile § 20-26 Vor-

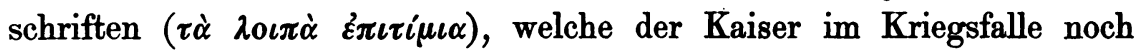
aufserdem den aufgestellten Truppen zu verkünden für nötig hält. Diese nachträglichen Bestimmungen zeichnen sich durch anderen Stil aus und insbesondere durch das in jeder derselben wiederkehrende $x \varepsilon \lambda \varepsilon v_{0} \mu \varepsilon \nu$. 


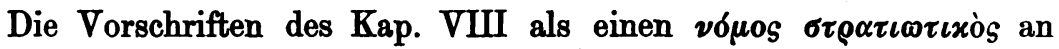

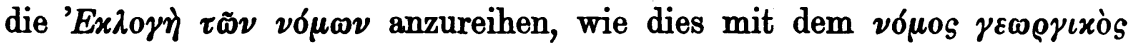

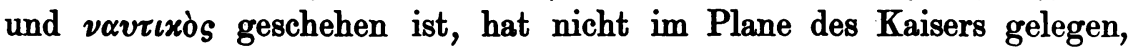
vielleicht weil derselbe von anderen Gerichten, den militärischen, zu gebrauchen war. Private dagegen haben daraus Veranlassung genommen, ein Militärgesetz zu componiren, welches in verschiedenen Recensionen in den Anhängen der Ecloge und ihrer verschiedenen $\mathrm{Be}$ arbeitungen erscheint.

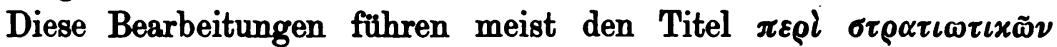

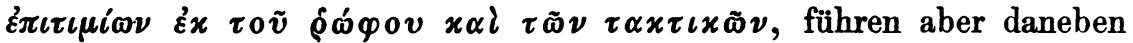
auch noch Digesten und Codex als Quellen an. Zu Kap. VIII der Tactica Leonis stehen sie in gleichem Verhältnis, nur mit dem Unterschiede, dals der zweite Teil der Strafbestimmungen des Kap. VIII in wesentlich gleichem Wortlaut und in gleicher Aufeinanderfolge, die Strafbestimmungen des ersten Teils aber mit bedeutenden Varianten und in verschiedener Aufeinanderfolge, auch untermischt mit Stellen aus anderen Quellen wiedergegeben werden.

Grofskmehlen.

Zachariä v. Lingenthal.

Bemerkung der Redaktion: Im April dieses Jahres stellte Herr Geheimrat Dr. K. E. Zachariä von Lingenthal der Byz. Z. eine Abhandlung über

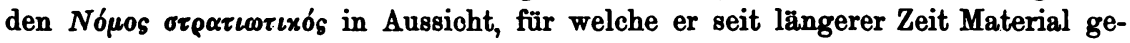
sammelt hatte. Ein böses Geschick hat die Vollendung dieser einen so wichtigen Gegenstand betreffenden Studie leider zunächst verhindert. Am 7. Mai erhielt ich den oben mitgeteilten Entwurf mit folgenden Zeilen, welche über die Unterbrechung der Arbeit und zugleich über den Plan derselben aufklären: „Die Hornhauttrübung meiner Augen ist so weit vorgeschritten, dafs ich kaum mehr das Notdürftigste lesen und schreiben kann, auf gelehrte Arbeiten aber verzichten mufs. Von dem Aufsatz, welchen ich für Sie schreiben wollte, lege ich die drei ersten Blätter bei. In weiterer Folge sollte der Aufsatz die drei verschiedenen Rezensionen der Militärgesetze, wie sie in Handschriften und Ausgaben erkennbar sind, und die drei Doppelstellen, die in denselben vorkommen, behandeln. So sollte der Rufus (bei \& 32 Leuncl.) umstellt und eingefangen werden. Ich gebe Ihnen anheim, ob Sie mit dem Anfange des. Aufsatzes und diesen Schlufsandeutungen etwas anfangen können und wollen, und verabschiede mich als Ihr ergebener Dr. K. E. Zachariä von Lingenthal." Möge es dem hochverdienten Begründer der Geschichte des byzantinischen Rechtes vergönnt sein, seine Arbeiten wieder aufzunehmen und unserer Disziplin noch lange mit Rat und That beizustehen! 